\title{
Variações na composição isotópica de oxigênio na neve superficial ao longo de uma travessia antártica
}

\author{
Luciano MARQUETTO ${ }^{1}$, Jefferson C. SIMÕES ${ }^{1}$, Gino CASASSA ${ }^{2}$, Douglas S. INTRONE ${ }^{3}$ \& Elaine \\ Alves DOS SANTOS ${ }^{4}$
}

1 Centro Polar e Climático, Instituto de Geociências, Universidade Federal do Rio Grande do Sul. Av. Bento Gonçalves, 9500, CEP 91.540-000, Porto Alegre, RS, Brasil. E-mail: luciano.marquetto@gmail.com; jefferson.simoes@ufrgs.br.

2 Centro de Estudios Cientificos, Av. Arturo Prat 514, Casilla 1469, Valdivia, Chile. Los Aromos 3408, Las Vertientes, Santiago, Chile. E-mail: gino.casassa@gmail.com

3 Climate Change Institute, University of Maine, 303 Bryand Global Sciences Center, Orono, ME 04469-5790, EUA.

E-mail: introne@maine.edu.

4 Laboratório de Radioecologia e Mudanças Globais, Instituto de Biologia, Universidade do Estado do Rio de Janeiro. 20550 013 - Rio de Janeiro, RJ, Brasil. E-mail: elainealves1301@gmail.com.

Recebido em 05/2013. Aceito para publicação em 08/2015.

Versão online publicada em 11/12/2015 (www.pesquisasemgeociencias.ufrgs.br)

\begin{abstract}
Resumo - Este trabalho apresenta a variabilidade das razões de isótopos de oxigênio na neve superficial ao longo de uma travessia do manto de gelo da Antártida ocidental realizada no verão austral de 2004/2005 por uma expedição conjunta entre pesquisadores chilenos e brasileiros. A travessia foi realizada entre a estação chilena Tenente Parodi, em Patriot Hills $\left(80^{\circ} 18^{\prime} \mathrm{S}, 081^{\circ} 21^{\prime} \mathrm{W}\right)$ e o Polo Sul geográfico, cobrindo uma distância de mais de $1.205 \mathrm{~km}$. Foram coletadas amostras de neve superficial (entre 0,05 a $0,2 \mathrm{~m}$ de profundidade) aproximadamente a cada $10 \mathrm{~km}$ (total de 104 amostras). A temperatura média anual foi obtida em seis pontos espaçados aproximadamente $220 \mathrm{~km}$ entre si, a uma profundidade entre 10 e $15 \mathrm{~m}$. A razão isotópica do oxigênio $\left(\delta^{18} 0\right)$ de cada amostra foi determinada por espectrometria de massas com fonte de gás (GSMS - Gas Source Mass Spectrometry) com precisão de $0,05 \%$. Os resultados indicam forte correlação entre $\delta^{18} 0$ e temperatura local, latitude, altitude e distância da costa, sendo a primeira positiva e as outras negativas. Valores isotópicos relativamente altos são encontrados entre $87^{\circ} 30^{\prime} \mathrm{S}$ e $86^{\circ} 44^{\prime} \mathrm{S}$, resultantes de processos pós-deposicionais devido à formação de glaze ice (esmalte de gelo) identificadas em campo. 0 gradiente $\delta^{18} \mathrm{O} /$ Elevação encontrado foi de $-0,08 \%$ o $/ 100 \mathrm{~m}$ e o gradiente $\delta^{18} \mathrm{O} /$ Temperatura de $0,743 \% /{ }^{\circ} \mathrm{C}$. Com exceção das anomalias citadas, os resultados são concordantes com os encontrados por outros pesquisadores no manto de gelo antártico.

Palavras-chave: isótopos de oxigênio, neve superficial, Antártica.
\end{abstract}

Abstract - OXYGEN ISOTOPIC COMPOSITION VARIATIONS IN SURFACE SNOW ALONG AN ANTARCTIC TRAVERSE. This work presents the distribution of oxygen isotope ratios in the surface snow in the West Antarctic Ice Sheet during the Chilean-Brazilian traverse held in the austral summer of 2004/2005. The traverse was carried out from Chilean Antarctic station Tenente Parodi, in Patriot Hills $\left(80^{\circ} 18^{\prime} \mathrm{S}\right.$, $81^{\circ} 21^{\prime} \mathrm{W}$ ) and the Geographic South Pole, along more than $1.205 \mathrm{~km}$. We collected superficial snow samples of the upper 0.05 to $0.2 \mathrm{~m}$ deep, approximately at each $10 \mathrm{~km}$ (total of 104 samples). The average annual temperature was determined at six points spaced approximately $220 \mathrm{~km}$ apart, at a depth between 10 and $15 \mathrm{~m}$. The oxygen isotope ratio $\left(\delta^{18} 0\right)$ of each sample was determined by mass spectrometry with gas source (GSMS - Gas Source Mass Spectrometry) with a $0.05 \%$ precision. Results point out to the strong correlation between $\delta^{18} 0$ and the local temperature, latitude, altitude and distance from the coast, the first being positive and the others negative. Anomalous relatively high isotopic values are found between $87^{\circ} 30^{\prime} \mathrm{S}$ and $86^{\circ} 44^{\prime} \mathrm{S}$, which are associated to post-depositional processes due to formation of glaze ice as identified in the field. The gradient $\delta$ ${ }^{18} \mathrm{O} /$ Altitude is $-0.08 \%$ o $/ 100 \mathrm{~m}$ and the $\delta{ }^{18} \mathrm{O} / \mathrm{Temperature}$ is $0.743 \% 0 /{ }^{\circ} \mathrm{C}$. Excepting for the cited anomalies, results are consistent with those found by other researchers in the Antarctic ice sheet. Keywords: oxygen isotopes, superficial snow, Antarctica. 


\section{Introdução}

O estudo das variações da composição de isótopos estáveis na neve e no gelo é umas das principais ferramentas em Glaciologia. Desde a década de 1950, quando pesquisadores (Dansgaard, 1953; Epstein \& Mayeda, 1953; Craig, 1961) constataram a correlação positiva existente entre as razões de isótopos estáveis de oxigênio nas precipitações em altas e médias latitudes e a temperatura local, essas análises tem papel essencial nas pesquisas de testemunhos de gelo. A amostragem da neve tornou-se essencial para estudos paleoclimáticos (Masson-Delmotte et al., 2008) e glaciológicos (Cuffey \& Patterson, 2010) nos dois mantos de gelo que cobrem a Antártica e a Groenlândia.

Dansgaard (1954) ao estudar o potencial da análise dos isótopos estáveis de hidrogênio e oxigênio em amostras de neve, constatou que as proporções de isótopos mais pesados $\left(\mathrm{H}^{2}\right.$ e $\left.\mathrm{O}^{18}\right)$ decrescem com aumento de altitude e latitude. Epstein (1956) complementou essa descoberta ao mostrar que existe uma variação sazonal na composição de isótopos de oxigênio da neve, indicando que períodos mais frios produzem neve empobrecida do isótopo mais pesado.

Nas regiões polares a composição isotópica do hidrogênio e do oxigênio nas precipitações apresenta uma forte correlação com a temperatura local (Dansgaard et al., 1973; Lorius \& Merlivat, 1977) em especial com a temperatura de condensação da neve (Dansgaard, 1964). Essas relações foram e continuam sendo utilizadas na reconstrução do registro paleoclimático em testemunhos de gelo na Antártica e na Groelândia (Masson-Delmotte et al., 2008; Ding et al., 2010; Hou et al., 2012) e proveram os dois registros mais detalhados da variação da temperatura da atmosfera ao longo do Quaternário, aqueles do testemunho de gelo de Vostok (78 $28^{\circ} \mathrm{S}, 106^{\circ} 48^{\prime} \mathrm{E}$; $3.520 \mathrm{~m}$ de profundidade, últimos 420 mil anos; Petit et al. 1999) e do EPICA no Domo C $\left(75^{\circ} 07,32^{\prime}\right.$ S, $123^{\circ} 18,72^{\prime} \mathrm{E}$; $3.270 \mathrm{~m}$ de profundidade, últimos $800 \mathrm{mil}$ anos, EPICA Community Members, 2004).

No entanto, se observa que a relação entre as razões isotópicas de oxigênio na neve superficial e a temperatura em diferentes locais do continente antártico difere significativamente (Qin et al., 1994), de modo que estudos da distribuição geográfica das razões isotópicas são importantes para refinar os modelos paleoclimáticos existentes e para fornecer dados em escala adequada a estes.

Tendo como um de seus objetivos contribuir para um melhor detalhamento das razões isotópicas da Antártica Ocidental (parte do continente que está no hemisfério ocidental e é separado da Antártica Oriental pelas montanhas Transantárticas) foi realizada no verão de 2004-2005 uma travessia organizada pelo governo chileno em parceria com pesquisadores brasileiros (a travessia Chileno-Brasileiro antártica). 0 presente trabalho apresenta os resultados das medições da razão isotópica do oxigênio dessas amostras, reconstruindo a variabilidade espacial na superfície do manto de gelo antártico ao longo da travessia entre $80^{\circ}$ e $90^{\circ} \mathrm{S}$. Os resultados podem ser interpretados em termos de variações na temperatura de condensação da precipitação (Petit et al., 1999).

\section{Revisão sobre o tema}

\subsection{Fracionamento de isótopos estáveis de hidrogê- nio e oxigênio na precipitação}

Na natureza, os processos de fracionamento dos isótopos estáveis de hidrogênio e oxigênio que compõem a água são influenciados por fatores como a temperatura e a sazonalidade do clima, e também por fatores geográficos, como a altitude, latitude e continentalidade (avanço de determinada massa de ar continente adentro, se distanciando da costa) (Dansgaard, 1964).

Sob o ponto de vista hidrológico, a pressão de vapor superior do $\mathrm{H}_{2}{ }^{16} \mathrm{O}$ em comparação com as pressões de vapor de $\mathrm{HDO}$ e $\mathrm{H}_{2}{ }^{18} \mathrm{O}$ é destaca$\mathrm{da}$, pois este fator causa fracionamento em todos processos de condensação e também nos de evaporação da água na condição líquida (Dansgaard, 1964).

Se o processo de fracionamento ocorrer lentamente, a ponto de as condições de equilíbrio entre fases serem alcançadas, o fator de fracionamento $(\alpha)$ é a simples razão entre a pressão de vapor do componente leve $(p)$ e a pressão de vapor do componente pesado $\left(p^{\prime}\right)$ :

$$
\alpha=p / p^{\prime}
$$

Em temperaturas amenas $\left(\sim 20^{\circ} \mathrm{C}\right), \alpha($ HDO $)=$ 1,08 ; e $\alpha\left(\mathrm{H}_{2}{ }^{18} \mathrm{O}\right)=1,009$, de modo que o empobrecimento no vapor em equilíbrio com a água original é de aproximadamente $80 \%$ em ${ }^{18} 0$ e $9 \%$ em D (Dansgaard, 1964). Quando ocorre a condensação, devido à menor pressão de vapor do HDO (10\% menor) e $\mathrm{H}_{2}{ }^{18} \mathrm{O}$ ( $1 \%$ menor), essas duas moléculas passam para o estado líquido com maior facilidade do que a molécula de $\mathrm{H}_{2}{ }^{16} \mathrm{O}$.

Em condições de equilíbrio, a condensação pode ocorrer segundo as condições do modelo 
Rayleigh proposto por Dansgaard (1964). Esse processo ocorre de forma lenta, e gera imediata remoção do condensado após sua formação. 0 processo Rayleigh causa maior fracionamento do que aqueles onde as duas fases equilibram-se mutuamente por troca isotópica.

No entanto, nem sempre o fracionamento isotópico é causado por esses processos de equilíbrio simples. Em reações rápidas, o efeito cinético apresenta grande importância. Esse efeito ocorre porque a difusividade molecular do $\mathrm{HDO}$ e $\mathrm{H}_{2}{ }^{18} \mathrm{O}$ no ar é menor do que a do $\mathrm{H}_{2}{ }^{16} \mathrm{O}$, ou seja, o componente mais leve tem uma maior taxa de reação (c) do que os componentes pesados $\left[\mathrm{c}\left(\mathrm{H}_{2}{ }^{16} \mathrm{O}\right)>\mathrm{c}(\mathrm{HDO})>\right.$ $\left.\mathrm{c}\left(\mathrm{H}_{2}{ }^{18} \mathrm{O}\right)\right]$ (Dansgaard, 1964). Nesse mesmo artigo, Dansgaard relata experiências demonstrando que o deutério (D) é muito menos sensível aos efeitos cinéticos do que o ${ }^{18} \mathrm{O}$. Esse efeito é independente da temperatura e aplica-se a processos de evaporação e condensação que não ocorrem em equilíbrio (Jouzel et al., 1997). Masson-Delmotte et al. (2008), pela compilação de dados de mais de 1000 sítios, mostram que na neve antártica o fracionamento isotópico também varia de acordo com os fatores citados.

\subsection{Escala " $\delta$ " e o padrão SMOW}

Por convenção as razões isotópicas de oxigênio e hidrogênio (isótopo pesado sobre o leve, ou seja, ${ }^{18} \mathrm{O} /{ }^{16} \mathrm{O}$ e ${ }^{2} \mathrm{H} /{ }^{1} \mathrm{H}$ ) na água não são expressas em um valor absoluto, e sim em uma escala relativa $(\delta)$ a uma amostra padrão. Inicialmente a amostra padrão era um corpo d'água hipotético, definido com base em análises isotópicas de amostras de água retiradas em profundidades entre 500 e 2000 $\mathrm{m}$ em áreas de oceano aberto no Atlântico, Pacífico e Índico (Craig, 1961). Esta amostra era chamada de SMOW (Standard Mean Ocean Water) e era utilizada como ponto zero da escala $\delta$.

No entanto o termo SMOW passou a ser utilizado para diferentes amostras padrão sem um controle efetivo do valor isotópico destas, causando confusões nos resultados obtidos. Deste modo, a International Union of Pure and Aplied Chemistry (IUPAC) recomendou que o uso do padrão SMOW fosse descontinuado, e em seu lugar propôs o padrão VSMOW (Vienna Standard Mean Ocean Water), um padrão fabricado em laboratório e distribuído internacionalmente (Coplen, 1994; Hornberger, 1995).

Devido à eminente exaustão das amostras do padrão VSMOW, em 2006 a International Atomic Energy Agency (IAEA) lançou o padrão VSMOW2, idêntico ao primeiro. Os resultados de $\delta^{18} 0$ para amostras de água continuam sendo apresentados relativamente ao padrão VSMOW (IAEA, 2009). As razões isotópicas de nossas amostras foram normalizadas em relação à escala VSMOV e calibradas com os padrões SLAP (Standard Light Antarctic Precipitation) e GISP (Greenland Ice Sheet Precipitation) seguindo as recomendações da IAEA.

A fórmula que define a quantidade de isótopos estáveis em uma determinada amostra é expressa como uma diferença relativa entre a razão isotópica da amostra com relação à razão do padrão (VSMOW):

$$
\delta_{\text {amostra }}=\frac{R_{\text {amostra }}-R_{\text {VSMOW }}}{R_{\text {VSMOW }}} \times 1000
$$

Onde $\mathrm{R}_{\text {amostra }}$ é a razão entre ${ }^{18} \mathrm{O}$ e ${ }^{16} \mathrm{O}$ na amos-
tra (ou D e H);
$\mathrm{R}_{\mathrm{VSMow}}$ é a razão entre ${ }^{18} \mathrm{O}$ e ${ }^{16} \mathrm{O}$ (ou D e H) no
padrão internacional (VSMOW);
$\delta_{\text {amostra }}$ é a diferença da razão isotópica da amostra em relação ao VSMOW e expressa em partes por mil (\%o), pois as diferenças no conteúdo isotópico entre as amostras e a referência são muito pequenas.

Temperaturas obtidas no firn antártico (estágio intermediário entre a neve e o gelo, ainda permeável) foram utilizadas como indicadores da temperatura anual média dos sítios estudados, possibilitando compreender que nas regiões polares a composição isotópica do hidrogênio e do oxigênio nas precipitações apresenta uma forte correlação com a temperatura local (Dansgaard et al., 1973; Lorius \& Merlivat, 1977), em especial com a temperatura de condensação da neve (Dansgaard, 1964). Essas relações foram e continuam sendo utilizadas na reconstrução do registro paleoclimático em testemunhos de gelo na Antártica e na Groenlândia (Ding et al., 2010).

\section{3 Área, materiais e métodos}

No verão austral de 2004-2005 foi realizada pelo Chile a primeira travessia científica latino-americana de parte do manto de gelo antártico, quando então foram percorridos $2.411 \mathrm{~km}$ (ida e volta) a partir da estação chilena Tenente Parodi $\left(80^{\circ} 18^{\prime} \mathrm{S}, 081^{\circ} 21^{\prime} \mathrm{W} ; 700 \mathrm{~m}\right.$ de altitude) em Patriot Hills (Montanhas Ellsworth, Antártica Ocidental) até o Polo Sul geográfico $\left(90^{\circ} \mathrm{S}, 2.840 \mathrm{~m}\right.$ de altitude) usando um trator polar (Fig. 1). A equipe científica liderada G. Casassa do Centro de Estudios Cientificos de Valdivia contou com um brasileiro, J.C. Simões, o qual foi responsável pela amostragem superficial da neve e perfuração de testemu- 


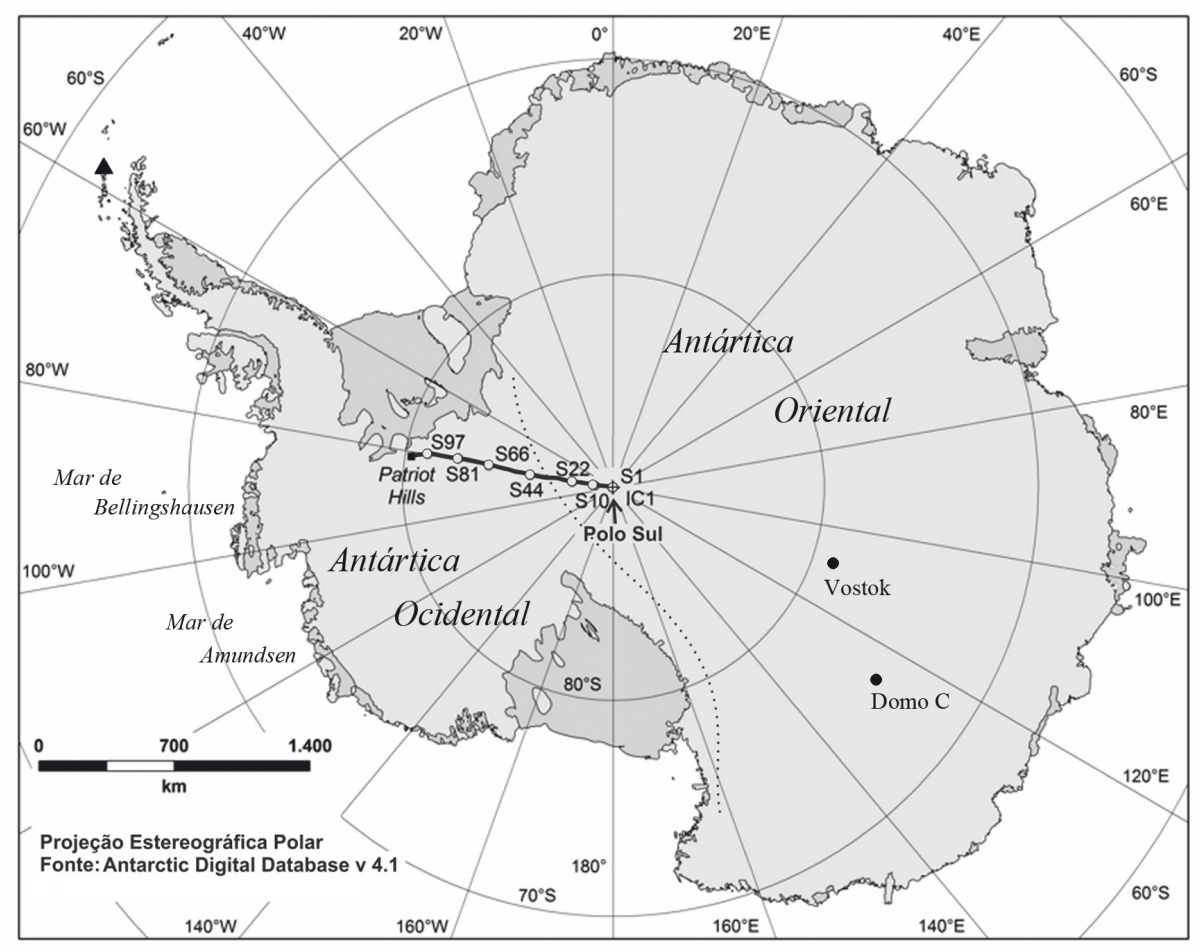

Figura 1. Travessia chileno-brasileira (em preto). Pontos brancos representam as seis perfurações realizadas no manto de gelo. A Estação Antártica Comandante Ferraz (EACF) é identificada por um triângulo no canto superior esquerdo do mapa. A linha pontilhada marca a posição aproximada das Montanhas Transantárticas.

nhos rasos de neve e firn ao longo do trajeto de retorno (1205,5 km). Os principais objetivos científicos dessa expedição foram: 1) coletar amostras de neve superficial para analisar a variabilidade espacial do conteúdo de isótopos estáveis de oxigênio (relatado neste artigo) e do conteúdo iônico; 2) coletar testemunhos de neve e firn que representem os últimos 150 a 200 anos da precipitação local (dependente da taxa anual de acumulação de neve no local da perfuração). No total foram amostrados seis testemunhos de até $46 \mathrm{~m}$ de profundidade, geralmente espaçados a cada $220 \mathrm{~km}$ (cerca de $2^{\circ}$ de latitude). Os resultados foram (i.e., Carlos et al., 2014; Marques et al., 2014) ou serão relatados em outras publicações. Ao longo da travessia também foram realizados levantamentos por radio ecossondagem do gelo na frequência de $150 \mathrm{MHz}$ para determinar a espessura e estrutura do manto de gelo (Casassa et al., 2005).

Essa travessia fez parte do programa ITASE (International Trans-Antarctic Scientific Expedition), idealizado em 1990 por pesquisadores de 12 países envolvidos em estudos de testemunhos de gelo (Mayewski \& Goodwin, 1997), cujo principal objetivo é entender como o ambiente atmosférico moderno (clima e composição atmosférica) está representado nas camadas superficiais do manto de gelo antártico (Mayewski et al., 2005). 0 foco central do programa são os últimos 200 anos do registro climático dos testemunhos de gelo. Atualmente o ITASE conta com uma série de dados resultantes de uma amostragem intensiva do manto de gelo antártico realizada por 20 países, de mais de duas centenas de testemunhos de neve e gelo ao longo de $20.000 \mathrm{~km}$ sobre o manto de gelo antártico.

\subsection{Trabalhos de campo e amostragem}

Especificamente, para a consecução do primeiro objetivo, tema deste trabalho, foi amostrado a neve superficial a cada $10 \mathrm{~km}$ de distância (Tab. 1) no trajeto de retorno da travessia. Primeiramente, 5 a $10 \mathrm{~cm}$ superficiais foram removidos utilizando uma pequena pá de plástico, seguido pela amostragem pressionando um vasilhame de polipropileno de $250 \mathrm{~mL}$ contra a superfície de neve. Ou seja, foram amostradas a neve superficial entre 5 a $20 \mathrm{~cm}$ de profundidade. 0 vasilhame foi previamente descontaminado com água ultrapura MilliQ (tal procedimento foi necessário porque a amostra também seria usada para determinação do conteúdo iônico por cromatografia líquida; ).

No total 104 amostras superficiais (Tab. 1) foram coletadas, os vasilhames colocados em caixas de isopor de alta densidade e mantidos no estado sólido durante todo o transporte. Na Antártica a temperatura não ultrapassou $-15^{\circ} \mathrm{C}$ durante toda a 
travessia, e de Patriot Hills até Boston as amostras mento do derretimento (à temperatura ambiente) foram mantidas frigorificadas. 0 transporte entre e preparação em laboratório, evitando assim qualBoston e Bangor, Maine, também foi realizado em quer fracionamento isotópico. caminhão frigorificado. Nesta cidade as amostras foram mantidas em câmara fria $\left(-20^{\circ} \mathrm{C}\right)$ até o mo-

Tabela 1. Dados das 104 amostras de neve superficial (primeiros $20 \mathrm{~cm}$ ) coletadas ao longo da travessia entre o Polo Sul geográfico e Patriot Hills. A posição geográfica das amostras foi determinada por GPS portátil com erro estimado de $\pm 30 \mathrm{~m}$, o erro da altitude é de $\pm 75 \mathrm{~m}$. A distância da costa foi calculada a partir da costa do mar de Amundsen-Bellingshausen. Temperatura (T) refere-se à temperatura média anual na superfície do local de amostragem. As concentrações isotópicas são expressas em termos de $(\% 0)$ normalizadas em relação a escala VSMOV. Note que não existem medições das razões isotópicas para as amostras S68, S100 e S105 (foram destruídas durante a subamostragem).

\begin{tabular}{|c|c|c|c|c|c|c|c|c|c|c|c|c|c|}
\hline Ponto & Lat (S) & Long (W) & $\begin{array}{l}\text { Altitude } \\
\text { (m) }\end{array}$ & $\begin{array}{c}\text { Distância } \\
\text { da } \\
\text { costa } \\
(\mathrm{km}) \\
\end{array}$ & $\mathrm{T}\left({ }^{\circ} \mathrm{C}\right)$ & $\begin{array}{l}\delta^{18} 0 \\
(\% 0)\end{array}$ & Ponto & Lat (S) & Long (W) & $\begin{array}{l}\text { Altitude } \\
\text { (m) }\end{array}$ & $\begin{array}{c}\text { Distância } \\
\text { da } \\
\text { costa } \\
(\mathrm{km}) \\
\end{array}$ & $\mathrm{T}\left({ }^{\circ} \mathrm{C}\right)$ & $\begin{array}{l}\delta^{18} 0 \\
(\% 0)\end{array}$ \\
\hline IC1 & $89^{\circ} 58^{\prime} 27,7^{\prime \prime}$ & $28^{\circ} 09^{\prime} 53,9^{\prime \prime}$ & 2833 & 2110 & -49 & & S53 & $85^{\circ} 10^{\prime} 27,4^{\prime \prime}$ & $80^{\circ} 52^{\prime} 03,6^{\prime \prime}$ & 1370 & 1772 & & $-42,5$ \\
\hline S1 & $89^{\circ} 57^{\prime} 00,0^{\prime \prime}$ & $93^{\circ} 09^{\prime} 38,7^{\prime \prime}$ & 2830 & 2109 & & $-51,6$ & S54 & $85^{\circ} 04^{\prime} 59,2^{\prime \prime}$ & $80^{\circ} 47^{\prime} 27,4^{\prime \prime}$ & 1360 & 1765 & & $-40,1$ \\
\hline S2 & $89^{\circ} 51^{\prime} 36,2^{\prime \prime}$ & $93^{\circ} 09^{\prime} 38,7^{\prime \prime}$ & 2825 & 2104 & & $-49,5$ & S55 & $84^{\circ} 59^{\prime} 28,0^{\prime \prime}$ & $80^{\circ} 43^{\prime} 28,9^{\prime \prime}$ & 1350 & 1759 & & $-38,4$ \\
\hline S3 & $89^{\circ} 46^{\prime} 04,2^{\prime \prime}$ & $88^{\circ} 30^{\prime} 16,3^{\prime \prime}$ & 2820 & 2097 & & $-49,6$ & S56 & $84^{\circ} 54^{\prime} 00,1^{\prime \prime}$ & $80^{\circ} 38^{\prime} 14,2^{\prime \prime}$ & 1341 & 1753 & & $-40,1$ \\
\hline S4 & $89^{\circ} 40^{\prime} 40,7^{\prime \prime}$ & $86^{\circ} 30^{\prime} 16,4^{\prime \prime}$ & 2810 & 2090 & & $-52,6$ & S57 & $84^{\circ} 48^{\prime} 17,6^{\prime \prime}$ & $80^{\circ} 37^{\prime} 38,4^{\prime \prime}$ & 1350 & 1747 & & $-39,1$ \\
\hline S5 & $89^{\circ} 35^{\prime} 23,9^{\prime \prime}$ & $85^{\circ} 08^{\prime} 33,5^{\prime \prime}$ & 2805 & 2083 & & $-48,1$ & S58 & $84^{\circ} 42^{\prime} 56,5^{\prime \prime}$ & $80^{\circ} 29^{\prime} 50,6^{\prime \prime}$ & 1300 & 1741 & & $-38,2$ \\
\hline S6 & $89^{\circ} 29^{\prime} 31,2^{\prime \prime}$ & $84^{\circ} 15^{\prime} 23,1^{\prime \prime}$ & 2789 & 2075 & & $-50,1$ & S59 & $84^{\circ} 36^{\prime} 28,0^{\prime \prime}$ & $80^{\circ} 26^{\prime} 01,2^{\prime \prime}$ & 1280 & 1734 & & $-38,0$ \\
\hline S7 & $89^{\circ} 24^{\prime} 03,6^{\prime \prime}$ & $83^{\circ} 52^{\prime} 37,9^{\prime \prime}$ & 2772 & 2068 & & $-49,8$ & $S 60$ & $84^{\circ} 32^{\prime} 00,1^{\prime \prime}$ & $80^{\circ} 23^{\prime} 30,8^{\prime \prime}$ & 1290 & 1729 & & $-39,2$ \\
\hline S8 & $89^{\circ} 18^{\prime} 38,7^{\prime \prime}$ & $83^{\circ} 32^{\prime} 09,2^{\prime \prime}$ & 2776 & 2062 & & $-49,4$ & S61 & $84^{\circ} 26^{\prime} 22,1^{\prime \prime}$ & $80^{\circ} 19^{\prime} 45,7^{\prime \prime}$ & 1268 & 1723 & & $-43,5$ \\
\hline S9 & $89^{\circ} 13^{\prime} 04,2^{\prime \prime}$ & $83^{\circ} 14^{\prime} 13,7^{\prime \prime}$ & 2771 & 2054 & & $-47,4$ & $S 62$ & $84^{\circ} 20^{\prime} 58,0^{\prime \prime}$ & $80^{\circ} 17^{\prime} 32,0^{\prime \prime}$ & 1282 & 1717 & & $-38,0$ \\
\hline S10 & $89^{\circ} 07^{\prime} 33,1^{\prime \prime}$ & $83^{\circ} 04^{\prime} 20,2^{\prime \prime}$ & 2774 & 2048 & & $-48,5$ & S63 & $84^{\circ} 15^{\prime} 24,7^{\prime \prime}$ & $80^{\circ} 15^{\prime} 41,1^{\prime \prime}$ & 1312 & 1712 & & $-36,0$ \\
\hline S11 & $89^{\circ} 01^{\prime} 59,0^{\prime \prime}$ & $82^{\circ} 49^{\prime} 15,0^{\prime \prime}$ & 2732 & 2041 & & $-51,5$ & S64 & $84^{\circ} 10^{\prime} 00,3^{\prime \prime}$ & $80^{\circ} 12^{\prime} 32,8^{\prime \prime}$ & 1300 & 1706 & & $-38,7$ \\
\hline S12 & $88^{\circ} 56^{\prime} 00,0^{\prime \prime}$ & $82^{\circ} 49^{\prime} 00,0^{\prime \prime}$ & 2770 & 2033 & & $-50,5$ & S65 & $84^{\circ} 04^{\prime} 28,9^{\prime \prime}$ & $80^{\circ} 10^{\prime} 06,7^{\prime \prime}$ & 1300 & 1700 & & $-41,3$ \\
\hline S13 & $88^{\circ} 50^{\prime} 53,2^{\prime \prime}$ & $82^{\circ} 38^{\prime} 39,8^{\prime \prime}$ & 2760 & 2027 & & $-49,0$ & S66 & $83^{\circ} 58^{\prime} 59,4^{\prime \prime}$ & $80^{\circ} 07^{\prime} 01,4^{\prime \prime}$ & 1295 & 1694 & -33 & $-41,8$ \\
\hline S14 & $88^{\circ} 45^{\prime} 31,2^{\prime \prime}$ & $82^{\circ} 33^{\prime} 05,7^{\prime \prime}$ & 2758 & 2020 & & $-51,4$ & S67 & $83^{\circ} 53^{\prime} 21,6^{\prime \prime}$ & $80^{\circ} 08^{\prime} 20,5^{\prime \prime}$ & 1300 & 1689 & & $-39,9$ \\
\hline S15 & $88^{\circ} 39^{\prime} 58,9^{\prime \prime}$ & $82^{\circ} 25^{\prime} 38,0^{\prime \prime}$ & 2744 & 2013 & & $-49,7$ & S68 & $83^{\circ} 47^{\prime} 58,6^{\prime \prime}$ & $80^{\circ} 05^{\prime} 33,8^{\prime \prime}$ & 1280 & 1684 & & \\
\hline S16 & $88^{\circ} 34^{\prime} 28,5^{\prime \prime}$ & $82^{\circ} 23^{\prime} 15,9^{\prime \prime}$ & 2750 & 2007 & & $-46,6$ & S69 & $83^{\circ} 42^{\prime} 19,1^{\prime \prime}$ & $80^{\circ} 06^{\prime} 57,0^{\prime \prime}$ & 1250 & 1679 & & $-36,6$ \\
\hline S17 & $88^{\circ} 28^{\prime} 44,4^{\prime \prime}$ & $82^{\circ} 15^{\prime} 42,5^{\prime \prime}$ & 2733 & 1999 & & $-49,7$ & S70 & $83^{\circ} 36^{\prime} 55,0^{\prime \prime}$ & $80^{\circ} 04^{\prime} 12,4$ & 1250 & 1673 & & $-42,5$ \\
\hline S18 & $88^{\circ} 23^{\prime} 05,5^{\prime \prime}$ & $82^{\circ} 12^{\prime} 15,6^{\prime \prime}$ & 2713 & 1993 & & $-48,2$ & S71 & $83^{\circ} 31^{\prime} 20,7^{\prime \prime}$ & $80^{\circ} 04^{\prime} 53,5$ & 1230 & 1668 & & $-36,4$ \\
\hline S19 & $88^{\circ} 17^{\prime} 41,2^{\prime \prime}$ & $82^{\circ} 09^{\prime} 40,0^{\prime \prime}$ & 2677 & 1986 & & $-49,1$ & S72 & $83^{\circ} 25^{\prime} 56,4^{\prime \prime}$ & $80^{\circ} 03^{\prime} 10,5^{\prime \prime}$ & 1190 & 1663 & & $-35,9$ \\
\hline S20 & $88^{\circ} 12^{\prime} 23,4^{\prime \prime}$ & $82^{\circ} 09^{\prime} 44,3$ & 2660 & 1980 & & $-40,2$ & S73 & $83^{\circ} 20^{\prime} 21,6^{\prime \prime}$ & $80^{\circ} 04^{\prime} 26,9^{\prime \prime}$ & 1150 & 1658 & & $-37,0$ \\
\hline S21 & $88^{\circ} 06^{\prime} 45,3^{\prime \prime}$ & $82^{\circ} 09^{\prime} 59,1^{\prime \prime}$ & 2623 & 1973 & & $-50,9$ & S74 & $83^{\circ} 14^{\prime} 56,3^{\prime \prime}$ & $80^{\circ} 02^{\prime} 15,3^{\prime \prime}$ & 1150 & 1653 & & $-35,1$ \\
\hline S22 & $88^{\circ} 01^{\prime} 21,3^{\prime \prime}$ & $82^{\circ} 04^{\prime} 21,7^{\prime \prime}$ & 2621 & 1967 & -45 & $-49,1$ & S75 & $83^{\circ} 08^{\prime} 44,4^{\prime \prime}$ & $80^{\circ} 00^{\prime} 40,0^{\prime \prime}$ & 1150 & 1647 & & $-36,5$ \\
\hline S23 & $87^{\circ} 53^{\prime} 08,9^{\prime \prime}$ & $82^{\circ} 01^{\prime} 53,0^{\prime \prime}$ & 2545 & 1957 & & $-44,2$ & S76 & $82^{\circ} 57^{\prime} 33,8^{\prime \prime}$ & $79^{\circ} 50^{\prime} 22,8^{\prime \prime}$ & 1050 & 1635 & & $-35,0$ \\
\hline S24 & $87^{\circ} 50^{\prime} 18,0^{\prime \prime}$ & $81^{\circ} 59^{\prime} 49,3^{\prime \prime}$ & 2525 & 1953 & & $-49,2$ & S77 & $82^{\circ} 52^{\prime} 27,5^{\prime \prime}$ & $79^{\circ} 45^{\prime} 59,8^{\prime \prime}$ & 1060 & 1630 & & $-38,1$ \\
\hline S25 & $87^{\circ} 43^{\prime} 41,3^{\prime \prime}$ & $81^{\circ} 52^{\prime} 52,5^{\prime \prime}$ & 2512 & 1945 & & $-45,0$ & S78 & $82^{\circ} 46^{\prime} 47,7^{\prime \prime}$ & $79^{\circ} 41^{\prime} 04,2^{\prime \prime}$ & 1020 & 1624 & & $-36,2$ \\
\hline S26 & $87^{\circ} 37^{\prime} 16,5^{\prime \prime}$ & $81^{\circ} 53^{\prime} 45,5^{\prime \prime}$ & 2460 & 1938 & & $-46,4$ & S79 & $82^{\circ} 41^{\prime} 26,1^{\prime \prime}$ & $79^{\circ} 36^{\prime} 21,0^{\prime \prime}$ & 1030 & 1619 & & $-34,7$ \\
\hline S27 & $87^{\circ} 30^{\prime} 45,6^{\prime \prime}$ & $82^{\circ} 24^{\prime} 34,7^{\prime \prime}$ & 2429 & 1932 & & $-39,6$ & S80 & $82^{\circ} 35^{\prime} 57,0^{\prime \prime}$ & $79^{\circ} 31^{\prime} 31,1^{\prime \prime}$ & 1000 & 1613 & & $-36,9$ \\
\hline S28 & $87^{\circ} 28^{\prime} 15,2^{\prime \prime}$ & $81^{\circ} 52^{\prime} 43,7^{\prime \prime}$ & 2385 & 1927 & & $-38,7$ & S81 & $82^{\circ} 30^{\prime} 30,8^{\prime \prime}$ & $79^{\circ} 28^{\prime} 02,7^{\prime \prime}$ & 950 & 1608 & -29 & $-39,6$ \\
\hline S29 & $87^{\circ} 22^{\prime} 34,4^{\prime \prime}$ & $81^{\circ} 58^{\prime} 01,9^{\prime \prime}$ & 2340 & 1921 & & $-38,2$ & S82 & $82^{\circ} 24^{\prime} 58,3^{\prime \prime}$ & $79^{\circ} 23^{\prime} 30,1^{\prime \prime}$ & 940 & 1602 & & $-32,6$ \\
\hline S30 & $87^{\circ} 17^{\prime} 12,3^{\prime \prime}$ & $81^{\circ} 49^{\prime} 08,8^{\prime \prime}$ & 2300 & 1915 & & $-42,4$ & 583 & $82^{\circ} 19^{\prime} 42,1^{\prime \prime}$ & $79^{\circ} 31^{\prime} 23,3^{\prime \prime}$ & 870 & 1599 & & $-41,8$ \\
\hline S31 & $87^{\circ} 11^{\prime} 32,9^{\prime \prime}$ & $81^{\circ} 44^{\prime} 58,3$ & 2300 & 1908 & & $-41,1$ & S84 & $82^{\circ} 14^{\prime} 12,9^{\prime \prime}$ & $79^{\circ} 40^{\prime} 33,9^{\prime \prime}$ & 850 & 1597 & & $-33,1$ \\
\hline S32 & $87^{\circ} 06^{\prime} 08,8$ & $81^{\circ} 45^{\prime} 50,5^{\prime \prime}$ & 2200 & 1902 & & $-39,6$ & S85 & $82^{\circ} 08^{\prime} 57,9^{\prime \prime}$ & $79^{\circ} 49^{\prime} 38,8^{\prime \prime}$ & 850 & 1595 & & $-30,0$ \\
\hline S33 & $87^{\circ} 00^{\prime} 20,5^{\prime \prime}$ & $81^{\circ} 44^{\prime} 30,3^{\prime \prime}$ & 2170 & 1895 & & $-39,6$ & S86 & $82^{\circ} 04^{\prime} 27,8^{\prime \prime}$ & $79^{\circ} 56^{\prime} 24,4^{\prime \prime}$ & 830 & 1593 & & $-33,7$ \\
\hline S34 & $86^{\circ} 55^{\prime} 07,4^{\prime \prime}$ & $81^{\circ} 44^{\prime} 26,9^{\prime \prime}$ & 2070 & 1889 & & $-36,9$ & S87 & $82^{\circ} 00^{\prime} 01,5^{\prime \prime}$ & $79^{\circ} 57^{\prime} 45,4^{\prime \prime}$ & 830 & 1589 & & $-33,5$ \\
\hline S35 & $86^{\circ} 49^{\prime} 33,1^{\prime \prime}$ & $81^{\circ} 45^{\prime} 19,1^{\prime \prime}$ & 1990 & 1883 & & $-38,3$ & S88 & $81^{\circ} 53^{\prime} 20,2^{\prime \prime}$ & $79^{\circ} 57^{\prime} 31,5^{\prime \prime}$ & 830 & 1584 & & $-35,7$ \\
\hline S36 & $86^{\circ} 44^{\prime} 08,2^{\prime \prime}$ & $81^{\circ} 42^{\prime} 06,7^{\prime \prime}$ & 1914 & 1877 & & $-34,7$ & S89 & $81^{\circ} 47^{\prime} 06,5^{\prime \prime}$ & $79^{\circ} 56^{\prime} 13,3^{\prime \prime}$ & 800 & 1579 & & $-32,7$ \\
\hline S37 & $86^{\circ} 38^{\prime} 30,7^{\prime \prime}$ & $81^{\circ} 40^{\prime} 25,3^{\prime \prime}$ & 1900 & 1870 & & $-41,6$ & S90 & $81^{\circ} 41^{\prime} 37,4^{\prime \prime}$ & $79^{\circ} 56^{\prime} 04,9^{\prime \prime}$ & 800 & 1575 & & $-34,3$ \\
\hline S38 & $86^{\circ} 33^{\prime} 05,9^{\prime \prime}$ & $81^{\circ} 40^{\prime} 58,6^{\prime \prime}$ & 1860 & 1865 & & $-45,0$ & S91 & $81^{\circ} 36^{\prime} 07,9^{\prime \prime}$ & $79^{\circ} 54^{\prime} 48,8^{\prime \prime}$ & 730 & 1571 & & $-35,4$ \\
\hline S39 & $86^{\circ} 27^{\prime} 31,2^{\prime \prime}$ & $81^{\circ} 39^{\prime} 12,1^{\prime \prime}$ & 1840 & 1858 & & $-43,9$ & S92 & $81^{\circ} 30^{\prime} 34,7^{\prime \prime}$ & $79^{\circ} 54^{\prime} 34,1^{\prime \prime}$ & 710 & 1566 & & $-31,8$ \\
\hline S40 & $86^{\circ} 22^{\prime} 01,2^{\prime \prime}$ & $81^{\circ} 38^{\prime} 13,1^{\prime \prime}$ & 1802 & 1852 & & $-44,7$ & S93 & $81^{\circ} 25^{\prime} 06,4^{\prime \prime}$ & $79^{\circ} 53^{\prime} 46,9^{\prime \prime}$ & 700 & 1562 & & $-31,6$ \\
\hline S41 & $86^{\circ} 16^{\prime} 35,0^{\prime \prime}$ & $81^{\circ} 37^{\prime} 53,6^{\prime \prime}$ & 1754 & 1846 & & $-46,7$ & S94 & $81^{\circ} 19^{\prime} 34,5^{\prime \prime}$ & $79^{\circ} 53^{\prime} 17,1^{\prime \prime}$ & 700 & 1558 & & $-37,9$ \\
\hline S42 & $86^{\circ} 11^{\prime} 00,3^{\prime \prime}$ & $81^{\circ} 37^{\prime} 02,9^{\prime \prime}$ & 1740 & 1840 & & $-45,1$ & S95 & $81^{\circ} 14^{\prime} 09,1^{\prime \prime}$ & $79^{\circ} 52^{\prime} 07,6^{\prime \prime}$ & 730 & 1554 & & $-32,1$ \\
\hline$S 43$ & $86^{\circ} 05^{\prime} 25,7^{\prime \prime}$ & $81^{\circ} 36^{\prime} 08,4^{\prime \prime}$ & 1710 & 1834 & & $-43,2$ & S96 & $81^{\circ} 08^{\prime} 35,1^{\prime \prime}$ & $79^{\circ} 50^{\prime} 14,3 "$ & 720 & 1550 & & $-32,3$ \\
\hline 544 & $85^{\circ} 59^{\prime} 57,5^{\prime \prime}$ & $81^{\circ} 35^{\prime} 06,3^{\prime \prime}$ & 1620 & 1828 & -36 & $-43,2$ & S97 & $81^{\circ} 03^{\prime} 10,1^{\prime \prime}$ & $79^{\circ} 50^{\prime} 09,1^{\prime \prime}$ & 750 & 1546 & -27 & $-32,8$ \\
\hline S45 & $85^{\circ} 54^{\prime} 20,0^{\prime \prime}$ & $81^{\circ} 34^{\prime} 44,8^{\prime \prime}$ & 1590 & 1822 & & $-39,8$ & S98 & $80^{\circ} 57^{\prime} 37,0^{\prime \prime}$ & $79^{\circ} 49^{\prime} 16,2^{\prime \prime}$ & 750 & 1542 & & $-36,0$ \\
\hline S46 & $85^{\circ} 48^{\prime} 55,1^{\prime \prime}$ & $81^{\circ} 33^{\prime} 04,7^{\prime \prime}$ & 1570 & 1816 & & $-44,8$ & S99 & $80^{\circ} 42^{\prime} 13,1^{\prime \prime}$ & $79^{\circ} 48^{\prime} 18,8^{\prime \prime}$ & 750 & 1532 & & $-36,0$ \\
\hline S47 & $85^{\circ} 43^{\prime} 20,3^{\prime \prime}$ & $81^{\circ} 25^{\prime} 54,3^{\prime \prime}$ & 1550 & 1810 & & $-39,5$ & S100 & $80^{\circ} 46^{\prime} 44,1^{\prime \prime}$ & $79^{\circ} 47^{\prime} 08,7^{\prime \prime}$ & 750 & 1534 & & \\
\hline S48 & $85^{\circ} 37^{\prime} 57,0^{\prime \prime}$ & $81^{\circ} 19^{\prime} 30,8^{\prime \prime}$ & 1450 & 1803 & & $-41,0$ & S101 & $80^{\circ} 41^{\prime} 19,3^{\prime \prime}$ & $79^{\circ} 47^{\prime} 33,3^{\prime \prime}$ & 750 & 1531 & & $-31,7$ \\
\hline S49 & $85^{\circ} 31^{\prime} 23,8^{\prime \prime}$ & $81^{\circ} 11^{\prime} 45,6^{\prime \prime}$ & 1470 & 1796 & & $-45,0$ & S102 & $80^{\circ} 35^{\prime} 42,8^{\prime \prime}$ & $79^{\circ} 51^{\prime} 09,7^{\prime \prime}$ & 750 & 1528 & & $-31,7$ \\
\hline S50 & $85^{\circ} 25^{\prime} 59,6^{\prime \prime}$ & $81^{\circ} 09^{\prime} 46,9^{\prime \prime}$ & 1350 & 1790 & & $-46,0$ & S103 & $80^{\circ} 30^{\prime} 19,2^{\prime \prime}$ & $79^{\circ} 54^{\prime} 26,0^{\prime \prime}$ & 750 & 1526 & & $-33,5$ \\
\hline S51 & $85^{\circ} 20^{\prime} 57,1^{\prime \prime}$ & $81^{\circ} 02^{\prime} 16,9^{\prime \prime}$ & 1380 & 1784 & & $-42,2$ & S104 & $80^{\circ} 24^{\prime} 53,3^{\prime \prime}$ & $79^{\circ} 59^{\prime} 22,3^{\prime \prime}$ & 700 & 1524 & & $-33,5$ \\
\hline S52 & $85^{\circ} 15^{\prime} 57,4$ & $80^{\circ} 57^{\prime} 10,2$ & 1360 & 1778 & & $-42,5$ & S105 & $80^{\circ} 20^{\prime} 10,7^{\prime \prime}$ & $80^{\circ} 34^{\prime} 11,1^{\prime \prime}$ & 750 & 1531 & & \\
\hline
\end{tabular}


0 manto de gelo antártico cobre aproximadamente 13,6 milhões $\mathrm{km}^{2}$ e o número de estações meteorológicas ainda é muito restrito. Assim, a temperatura média do local de amostragem (essencial para determinar a relação entre o conteúdo de isótopos estáveis de uma amostra e a temperatura local) deve ser determinada de maneira indireta. Segundo Robin (1983), a temperatura nas camadas superiores das geleiras polares são governadas pela condutividade térmica da neve e do firn, variando sazonalmente. Dez metros abaixo da superfície, essas variações sazonais de temperatura são atenuadas e se aproximam da temperatura média anual da superfície da neve. Em profundidades maiores do que $15 \mathrm{~m}$, o controle da temperatura é feito pelo fluxo de calor geotérmico, pela quantidade de calor gerado por friç̧ão na base da geleira e pelo movimento diferencial interno (Cuffey \& Patterson, 2010). Deste modo, a temperatura da neve entre 10 e $15 \mathrm{~m}$ reflete mais fielmente a temperatura média do ar no sítio de amostragem. Nos seis locais de perfuração de testemunhos rasos (onde também foram realizadas amostragens da neve superficial) foi medida a temperatura entre 10 e $12 \mathrm{~m}$. Para isso utilizou-se uma sonda com resistência de prata previamente calibrada colocada na ponta de um cabo elétrico. A sonda permaneceu no poço de medição por aproximadamente $24 \mathrm{~h}$ para equilibrar a temperatura do ar contido no buraco do poço com aquela da neve/firn circundante.

A posição geográfica de cada ponto de amostragem foi determinada utilizando um GPS portátil com erro estimado máximo de $30 \mathrm{~m}$ e vertical de $75 \mathrm{~m}$.

\subsection{Técnicas analíticas}

O conteúdo isotópico $\left({ }^{18} \mathrm{O} /{ }^{16} \mathrm{O}\right)$ de cada amostra foi determinado por espectrometria de massa com fonte de gás (GSMS - Gas Source Mass Spectrometry) no Climate Change Institute, Universidade do Maine, Orono, Maine, EUA, seguindo os procedimentos analíticos descritos em Osterberg et al. (2006) para isótopos de oxigênio e são aqui reportados em termo de diferença de ${ }^{18} \mathrm{O} /{ }^{16} \mathrm{O}$ (i.e., $\delta^{18} \mathrm{O}$ ) do VSMOW (conforme descrito na seção 3). Usou-se técnica padrão de equilíbrio de $\mathrm{CO}_{2}$ gasoso, o gás foi então colocado em um equipamento Micromass Multiprep acoplado a um espectrômetro de massa de análise das razões de isótopos estáveis com precisão de $0,05 \%$ para $\delta^{18} 0$ (precisão baseada em análise estatística padrão de longo tempo).

0 modelo HYSPLIT (HYbrid Single-Particle Lagrangian Integrated Trajectory) (Draxler \& Rol- ph, 2003; Rolph, 2003) foi utilizado para calcular as trajetórias das parcelas de ar chegadas em seis pontos escolhidos ao longo da travessia (S1, S27, S36, S59, S80 e S105) durante o período do outono, época de maior precipitação na área (Monaghan et al., 2006). Esta etapa foi executada no Laboratório de Radioecologia e Mudanças Globais da Universidade do Estado do Rio de Janeiro (LARAMG-UERJ). Nesse modelo, as trajetórias são calculadas com base em um sistema híbrido entre as aproximações Euleriana e Lagrangiana. Os dados meteorológicos foram calculados a partir dos arquivos de reanálise NCAR/NCEP e podem ser encontrados no endereço ftp://arlftp.arlhq.noaa.gov/pub/archives/reanalysis. Os dados da trajetória foram produzidos no programa HYSPLIT (versão para Windows) e visualizados através do próprio Hysplit e também no ArcGis 9.0.

A saída do programa HYSPLIT/NOAA fornece um arquivo com extensão Gis para ser utilizado no programa ArcGIS. Esse arquivo possibilita fazer uma investigação mais detalhada do evento, tal como: determinar exatamente o caminho de uma dada massa de ar (por exemplo, posição, período e altura), inserir trajetórias diferentes em um mesmo mapa e mesclar outros dados. Para todas as retrotrajetórias rodadas, foram consideradas as coordenadas geográficas dos pontos de coleta e suas respectivas altitudes. 0 modelo utiliza dados meteorológicos do sistema de assimilação de dados globais (GDAS). 0 tempo de análise empregado na modelagem deste trabalho é de 10 dias (240 h), portanto os três pontos mensais fazem uma cobertura do transporte atmosférico mensal.

A distância da costa foi estimada a partir do ponto $71^{\circ} 04^{\prime} 54.98^{\prime \prime}$ e $82^{\circ} 53^{\prime} 56.55^{\prime \prime}$ W utilizando o programa ArcGis 10 e imagens de satélite da extensão mínima do gelo marinho no verão, entre os anos de 1979 e 2000, obtidas no NSIDC (National Snow and Ice Data Center, EUA).

\section{Resultados}

Na tabela 1 são apresentados os dados de coleta e os $\delta^{18} 0$ determinados para a neve superficial. Os valores de $\delta^{18} 0$ variam entre $-30,0 \%$ e $-52,6 \%$ (relativo ao VSMOW-SLAP) com média de $-40,1 \%$ e desvio padrão de 5,8 \%o. Foram gerados gráficos para examinar a relação entre o $\delta^{18} \mathrm{O}$ e a temperatura média anual na superfície (Fig. 2) e fatores geográficos (latitude, longitude, altitude e distância da costa; Fig. 3). As equações de regressão linear foram calculadas seguindo a rotina padrão do editor de planilhas MS Excel ${ }^{\odot}$ (Quadro 1): 


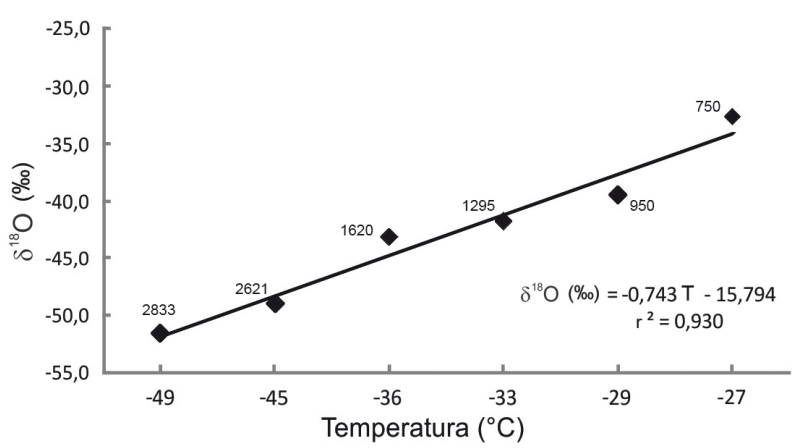

Figura 2. Relação entre $\delta^{18} \mathrm{O}$ na neve e a temperatura média anual ao longo da rota da travessia. Números pequenos juntos aos losangos são as altitudes (em metros) da amostragem.

0 cálculo das trajetórias das parcelas de ar mostra que o avanço predominante das precipitações sobre o continente antártico, nas determinadas datas, ocorreu entre os meridianos de $60^{\circ} \mathrm{e}$ $120^{\circ} \mathrm{W}$, pelos mares de Amundsen e Bellingshausen. Houve também avanço pelo mar de Weddell, entre os meridianos $-0^{\circ}$ e $60^{\circ} \mathrm{W}$ e pelo mar de Ross, entre $120^{\circ}$ e $150^{\circ} \mathrm{W}$, porém menos frequente (Figs. 4 e 5).

Com base nas trajetórias das parcelas de ar foi utilizada a região dos mares de Amundsen e de Bellingshausen como ponto de referência $\left(71^{\circ}\right.$ $04^{\prime} 54.98^{\prime \prime}$ e e $82^{\circ} 53^{\prime} 56.55^{\prime \prime} \mathrm{W}$ ) para a estimativa da distância da costa.

\section{Discussão}

\subsection{Relação entre $\delta^{18} \mathrm{O}$ e a temperatura média anual}

A variação do $\delta^{18} 0$ apresenta uma correlação positiva com a temperatura superficial (T) (Fig. 2), essa, evidentemente, diminui com a altitude. Apesar de terem sido mensurados dados em apenas seis pontos, o resultado $\left[\delta^{18} \mathrm{O}(\% 0)=0,743 \mathrm{~T}\left({ }^{\circ} \mathrm{C}\right)\right]$ encontra-se dentro do padrão obtido por outros autores (Qin et al., 1994; Masson-Delmotte et al., 2008; Wang \& Hou, 2009).

Qin et al. (1994) apontam para o fato de que em um modelo Rayleigh a altitude não é fator determinante da composição isotópica da precipitação, de modo que a redução do $\delta^{18} \mathrm{O}$ da precipitação observada na figura 3C também é resultado do decréscimo de temperatura e não do aumento da altitude em si.

\subsection{Variação espacial do $\delta^{18} \mathrm{O}$ ao longo do trajeto da travessia chileno-brasileira}

Ao longo do percurso de $1.205 \mathrm{~km}$ entre Patriot Hills (no setor ocidental do continente an- tártico) e o Polo Sul geográfico diversas variáveis podem afetar os valores das razões isotópicas de oxigênio, além dos fatores geográficos já citados. Efeitos pós-deposicionais como a ablação causada pelo vento ou a sublimação nas áreas de menor precipitação (Frezzotti et al., 2004; Neumann \& Waddington, 2004), bem como variações na taxa de acumulação anual, podem modificar a composição isotópica da neve originalmente precipitada.
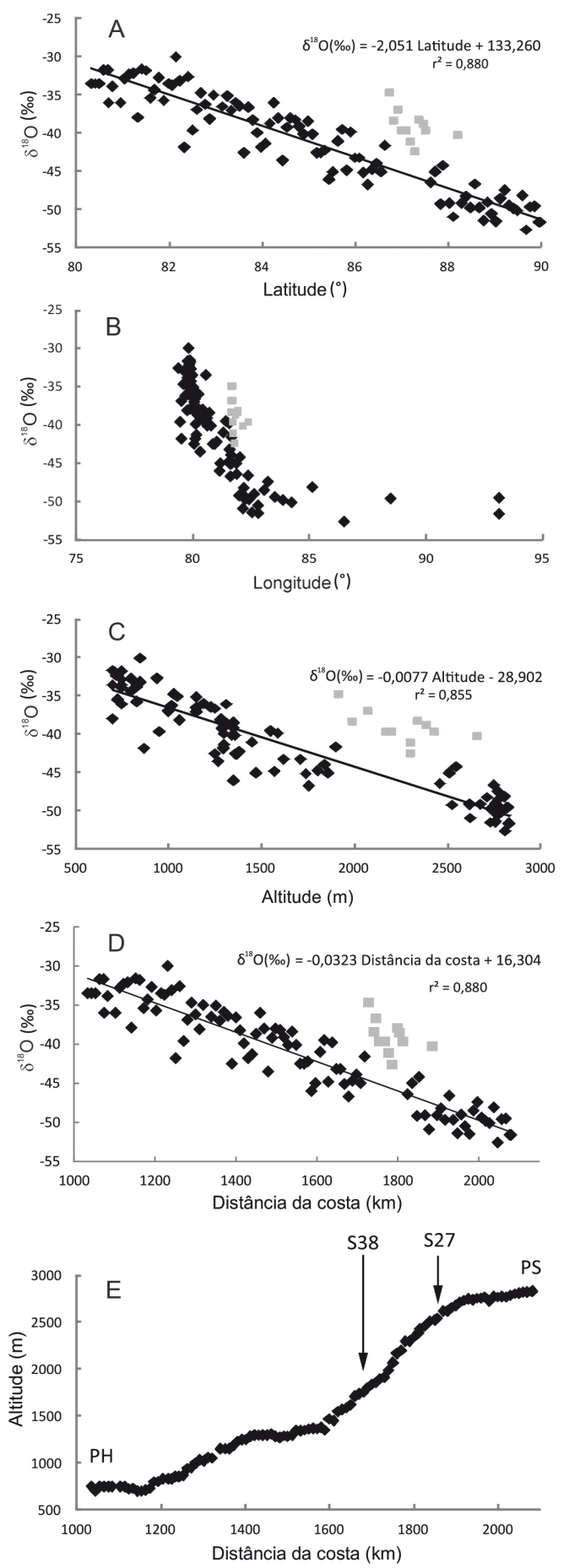

Figura 3. Relação entre $\delta^{18} 0$ e fatores geográficos. A) latitude; B) longitude; C) altitude; D) distância da costa; E) perfil altimétrico da travessia (Patriot Hills = PH; Polo Sul = PS). Nas figuras 3A-D quadrados cinza são pontos anômalos, identificados na figura $3 \mathrm{E}$ pelas setas com o número da amostra. 
Quadro 1. Equações de regressão linear indicando relação entre $\delta^{18} \mathrm{O}$ e temperatura, latitude, altitude e distância da costa.v

\begin{tabular}{|lll|}
\hline$\delta 180(\%)=0,743$ Temperatura $-15,794$ & sendo $r^{2}=0,930$ & $\mathrm{p}<0,02$ \\
\hline$\delta 180(\%)=-2,051$ Latitude $+133,260$ & sendo $r^{2}=0,880$ & $\mathrm{p}<0,0001$ \\
\hline$\delta 180(\%)=-0,0077$ Altitude $-28,902$ & sendo $r^{2}=0,855$ & $\mathrm{p}<0,0001$ \\
\hline$\delta 180(\%)=-0,0323$ Distância da costa $+16,304$ & sendo $^{2}=0,880$ & $\mathrm{p}<0,0001$ \\
\hline
\end{tabular}
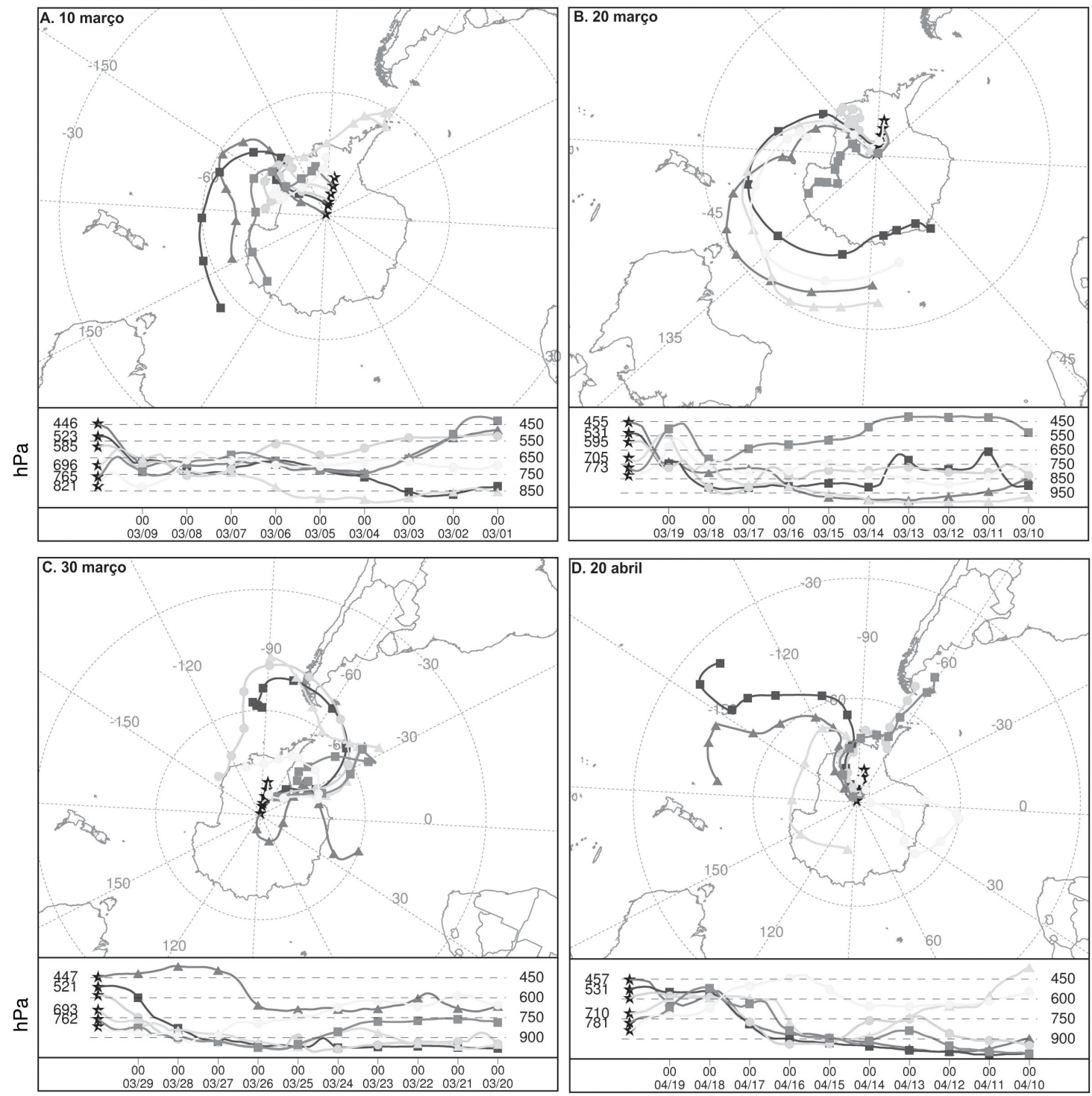

Figura 4. Trajetórias das massas de ar geradas pelo sistema HYSPLIT/NOAA. A) para a data de 10 de março; B) 20 de março; C) 30 de março; D) 10 de abril. Trajetórias de regressão finalizadas em 0000 UTC nas datas marcadas nos cantos superiores esquerdos de cada mapa.

É possível observar que o $\delta^{18} 0$ apresenta correlação negativa com fatores geográficos (latitude, longitude, distância da costa e altitude; fig. 3). A distância da costa foi calculada a partir dos mares de Amundsen e Bellingshausen, considerando que o cálculo das trajetórias das parcelas de ar indica que está é a origem principal das massas de ar que advectam sobre a rota de nossa travessia. 0 resultado mostra uma relação do $\left[\delta^{18} \mathrm{O}(\% 0)=-0,0323\right.$ Distância da costa $(\mathrm{km})]$, o que estaria dentro da faixa encontrada por Masson-Delmotte et al. (2008). A relação com a altitude é $\left[\delta^{18} \mathrm{O}(\% 0)=-0,0077\right.$
Elevação (m)] denota o efeito orográfico sobre as massas de ar que adentram o manto de gelo antártico. Já a relação do $\delta^{18} 0$ e a latitude $\left[\delta^{18} 0(\%)\right.$ $=-2,0357$ Latitude $\left(^{\circ}\right)$ ] é um simples artefato indireto da forma e posição do continente antártico, aproximadamente centrado no Polo Sul geográfico. Ou seja, ao adentrar o continente (e avançar para latitudes mais altas) as massas de ar sofrem um fracionamento isotópico intenso devido a menor temperatura, associado com o aumento da elevação e da distância da área fonte de vapor d'água.

0 gráfico relativo à longitude não apresenta 
uma relação linear entre esta e o $\delta^{18} 0$, no entanto sabe-se que o efeito da longitude não influi sobre as razões isotópicas (Dansgaard, 1964), sendo apenas um reflexo do fator continentalidade (ou distância da costa).
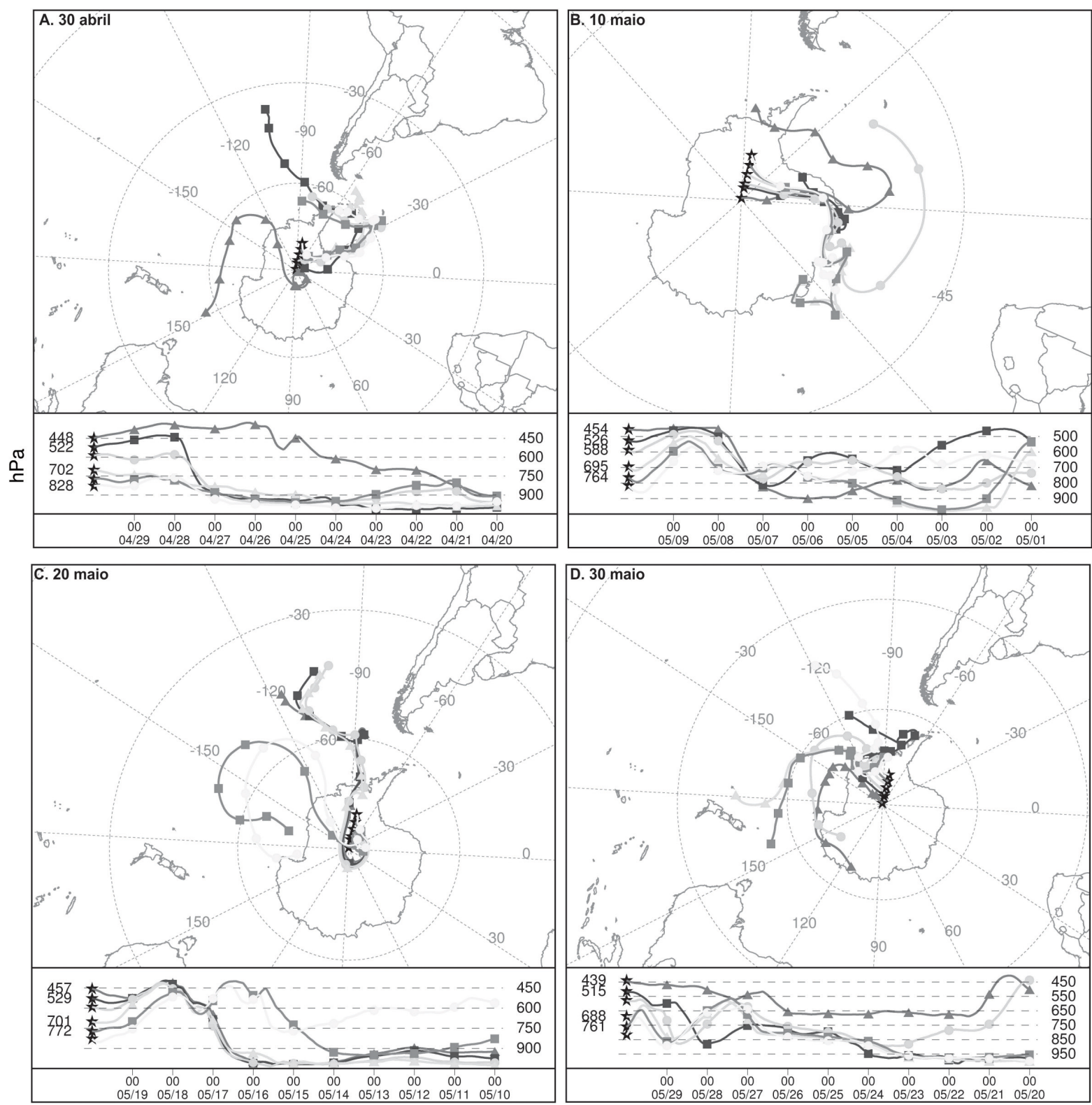

Figura 5. Trajetórias das massas de ar geradas pelo sistema HYSPLIT/NOAA. A) para a data de 30 de abril; B) 10 de maio; C) 20 de maio; D) 30 de maio. Trajetórias de regressão finalizadas em 0000 UTC nas datas marcadas nos cantos superiores esquerdos de cada mapa.

\subsection{Valores isotópicos anômalos entre $87^{\circ} 30^{\prime} e$ $86^{\circ} 44^{\prime} S$}

Nas figuras 3A, 3C e 3D percebe-se claramente uma anomalia na tendência do $\delta^{18} 0$ entre as amostras S38 $\left(86^{\circ} 33^{\prime} 05,9^{\prime \prime} \mathrm{S} ; 8^{\circ} 40^{\prime} 58,6^{\prime \prime} \mathrm{W}\right)$ e

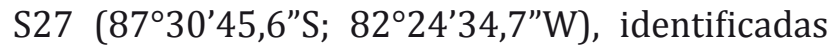
por quadrados cinza, e onde em menos de $20 \mathrm{~km}$ (entre os pontos S38 e S36, tab. 1) há um brusco aumento na proporção do isótopo mais pesado (o $\delta^{18} 0$ aumenta de $-45,0 \%$ para $-34,5 \% 0$ ), e aproximadamente $100 \mathrm{~km}$ adiante o $\delta^{18} 0$ retorna para a tendência de decréscimo com o aumento da al- titude e distância da linha de costa (Figs. 3C e 3D; Tab. 1).

Inicialmente essa anomalia foi atribuída ao efeito orográfico causado pelas Montanhas Transantárticas (Fig. 1), mas neste caso as razões isotópicas não voltariam a seguir a mesma tendência após passarem por aquela cadeia, pois ficariam muito mais pobres de ${ }^{18} \mathrm{O}$. 0 que se observa é um retorno aos valores esperados, pelas tendências de regressão mostradas nas figuras $3 \mathrm{~A}, 3 \mathrm{C}$ e $3 \mathrm{D}$. A população continuou a mesma, como se não houvesse uma área com valores anômalos.

Assim, considera-se que esta anomalia seja 
causada por um processo pós-deposicional atuando sobre a neve depositada, como sublimação ou ablação parcial do pacote de neve anual, este último por atuação eólica. Em ambos os casos, haveria uma remoção preferencial da neve superficial durante determinada estação do ano (Masson-Delmotte et al., 2008).

Bromwich et al. (2004) inferem que os efeitos da sublimação em áreas costeiras é muito maior do que em pontos localizados no interior do continente antártico, chegando até $40 \%$ do volume da precipitação. No entanto, ocorre sublimação mesmo em sítios nos quais há ventos fracos, como em Vostok e no Domo C no platô do manto de gelo antártico. Estima-se que a sublimação nesses pontos seja aproximadamente $10 \%$ da precipitação (Ekaykin et al., 2004; Frezzotti et al., 2004; Urbini et al., 2008). Frezzotti et al. (2004) estimam que apenas $10 \%$ da superfície do continente antártico apresente essa baixa intensidade de ventos, sendo o restante do manto de gelo afetado pelos efeitos de sublimação que variam entre $20 \%-75 \%$ do volume da precipitação.

No entanto, a sublimação não afeta a composição isotópica da neve residual, podendo, no máximo, causar um hiato sazonal pela remoção preferencial da neve, neste caso, da neve precipitada no inverno (e, portanto, com $\delta^{18} \mathrm{O}$ menor).

Ao examinar os dados da caderneta de campo, ficou claro que os pontos anômalos correspondem a áreas de glaze ice ("esmalte de gelo"). Essas áreas caracterizam-se por uma superfície de gelo polida pelo vento que recobre uma camada de firn recristalizado de granulometria grossa e são identificados em campo e imagens de satélite (Scambos et al., 2012; Dixon et al., 2013).

A área com valores isotópicos anômalos coincide com a parte mais íngreme do perfil da travessia (identificada na Fig. 3E). Em média, ao longo do trajeto o aclive é $2,4 \mathrm{~m} \mathrm{~km}^{-1}$, mas entre 1.865 e $1.877 \mathrm{~km}$ de linha de costa aumenta para $4,5 \mathrm{~m}$ $\mathrm{km}^{-1}$, isso indica que a área pode apresentar ventos catabáticos (ventos que transportam por gravidade o ar mais denso e frio do platô antártico para regiões mais baixas) velozes (esses ventos podem facilmente ultrapassar $70 \mathrm{~km} / \mathrm{h}$ ), forte evidência para corroborar a explicação sugerida para os altos valores relativos encontrados.

Por outro lado, é sabido que as superfícies polidas de gelo transmitem mais energia solar para o firn subjacente do que uma superfície de neve (Scambos et al., 2012). Isso aqueceria a subsuperfície, forçando um transporte de vapor d'água para cima, mudando a razão isotópica da camada superior de neve (Neumann \& Waddington, 2004).
Cabe ressaltar que na amostragem, a fina camada de gelo polido foi removida para que a neve (ou firn) pudesse ser coletada.

Os valores isotópicos relativamente altos encontrados entre $86^{\circ} 44^{\prime} \mathrm{S}$ e $87^{\circ} 30^{\prime}$ podem ser atribuídos tanto à remoção física de camadas de neve precipitada nos períodos mais frios do ano ou a um fracionamento pós-deposicional do pacote superficial de neve devido ao transporte de vapor d'água para cima.

\section{Conclusões}

É evidente a relação entre o $\delta^{18} O$ da neve superficial e a temperatura, altitude, latitude e a distância da costa. Como esperado, há uma tendência linear de decréscimo do $\delta^{18} 0$ conforme a amostragem se aproxima do Polo Sul Geográfico, partindo de $-32 \%$ (Patriot Hills, temperatura média de $-25^{\circ} \mathrm{C}$ ) para $-50 \%$ (Polo Sul geográfico, temperatura média de $-49^{\circ} \mathrm{C}$ ). Esses resultados estão de acordo com mapas de distribuição isotópica espacial obtidos por Qin et al. (1994) e Masson-Delmotte et al. (2008), que registraram razões isotópicas de -33 \%o e -30 \%o em Patriot Hills e -51 $\%$ e $-50 \%$ no Polo Sul Geográfico. 0 gradiente $\delta^{18} \mathrm{O} /$ elevação encontrado foi de $-0,08 \%$ \% $/ 100 \mathrm{~m}$ e o gradiente $\delta^{18} \mathrm{O} /$ temperatura foi de $0,743 \%$ o $/{ }^{\circ} \mathrm{C}$.

Os pontos nos quais as razões isotópicas se mostraram anômalas em relação à reta de tendência principal coincidem com áreas de glaze ice (esmalte de gelo) identificadas em campo, tratando-se, portanto, de alterações pós-deposicionais do $\delta^{18} \mathrm{O}$.

Agradecimentos - Este estudo é parte das investigações do Programa Antártico Brasileiro (PROANTAR) e foi financiado por projetos de pesquisa do Conselho Nacional de Desenvolvimento Científico e Tecnológico (CNPq), Processos 558117/2005-8 e 557053/20099. LM agradece ao CNPq por sua bolsa de mestrado. Os trabalhos de campo foram uma ação colaborativa do Centro de Estudios Científicos de Valdívia (Chile) e o Centro Polar e Climático da UFRGS, e contou com o apoio logístico do Exército do Chile e Força Aérea Chilena (FACh).

\section{Referências}

Bromwich, D.H., Guo, Z., Bai, L. \& Chen, Q. 2004. Modeled Antarctic Precipitation. Part I: Spatial and Temporal Variability. Journal of Climate, 17(3): 427-447.

Carlos, F.S., Simões, J.C., Handley, M. \& Casassa, G. 2014. Determinação de elementos-traço em testemunho de firn antártico usando ICP-MS. Geochimica Brasiliensis, 28(1): 97-107. 
Casassa, G., Urrutia, L., Antivil, M., Carrasco, P., Collao, G., Hernández, J., Iturrieta, C., Quinteros, J., Riveira, A., Simões, J.C., Teitelboim, C., Traub, R. \& Zamora, R. 2005. Primeira expedicion científica chilena aol Polo Sur. Boletín Antártico Chileno, 24(1): 30-34.

Coplen, T.B. 1994. Reporting of stable hydrogen, carbon, and oxygen isotopic abundances (Technical Report). Pure and Applied Chemistry, 66(2): 273-276.

Craig, H. 1961. Isotopic variations in meteoric waters. Science, 133(3465): 1702-1703.

Cuffey, K.M. \& Patterson, W. S. B. 2010. The Physics of Glaciers (4 ed.). Oxford, Academic Press, 710 p.

Dansgaard, W. 1953. The abundance of ${ }^{18} \mathrm{O}$ in atmospheric water and water vapour. Tellus, 5(4): 461-469.

Dansgaard, W. 1954. The ${ }^{18} 0$ abundance in fresh water. Geochimica et Cosmochimica Acta, 6(5-6): 241-260.

Dansgaard, W. 1964. Stable isotopes in precipitation. Tellus A, 16(4): 436-468.

Dansgaard, W., Johnsen, S.J. \& Clausen, H.B. 1973. Stable isotope glaciology. Meddelelser Grønland, 197: 1-53.

Ding, M.H., Xiao, C. De, Jin, B., Ren, J.W., Qin, D.H. \& Sun, W.Z. 2010. Distribution of $\delta{ }^{18} \mathrm{O}$ in surface snow along a transect from Zhongshan Station to Dome A, East Antarctica. Chinese Science Bulletin, 55(24): 2709-2714.

Dixon, D., Mayewski, P., Korotkikh, E., Sneed, S., Handley, M., Introne, D. \& Scambos, T. 2013. Variations in snow and firn chemistry along US ITASE traverses and the effect of surface glazing. The Cryosphere, 7: 515-535.

Draxler, R.R. \& Rolph, G.D. 2003. HYSPLIT (HYbrid Single Particle Lagrangian Integrated Trajectory) Model. NOAA Air Resources Laboratory, Silver Spring, Md. NOAA Air Resources Laboratory. Disponível em (http://www.arl.noaa.gov/ready/hysplit4.html). Acesso em 08/02/2013.

Ekaykin, A.A., Lipenkov, V.Y., Barkov, N.I., Kuzmina, I., Petit, J.R. ., Masson-Delmotte, V. \& Johnsen, S.J. 2004. The changes in isotope composition and accumulation of snow at Vostok Station, East Antarctica, over the past 200 years. Annals of Glaciology, 39: 569-575.

EPICA Community Members. 2004. Eight glacial cycles from an Antarctic ice core. Nature, 429(6992): 623-628.

Epstein, S. 1956. Variations of the ${ }^{18} \mathrm{O} /{ }^{16} \mathrm{O}$ ratios of fresh water and ice. Nuclear Science Series Report, 19: 20-28.

Epstein, S. \& Mayeda, T. 1953. Variations of ${ }^{18} \mathrm{O}$ content of waters from natural sources. Geochimica et Cosmochimica Acta, 4(5): 213-224.

Frezzotti, M., Pourchet, M., Flora, O., Gandolfi, S., Gay, M., Urbini, S., Vincent, C., Becagli, S., Gragnani, R., Proposito, M., Severi, M., Traversi, R., Udisti, R. \& Fily, M. 2004. New estimations of precipitation and surface sublimation in East Antarctica from snow accumulation measurements. Climate Dynamics, 23: 803-813.

Hornberger, G. 1995. New manuscript guidelines for the reporting of stable hydrogen, carbon, and oxygen isotope ratio data. Water Resources Research, 40(6): 1182.
Hou, S.G., Wang, Y.T. \& Pang, H.X. 2012. Climatology of stable isotopes in Antarctic snow and ice: Current status and prospects. Chinese Science Bulletin, 58(10): 1095-1106.

IAEA. International Atomic Energy Agency. 2009. Reference Sheet for VSMOW2 and SLAP2 international measurement standards. IAEA. Vienna: International Atomic Energy Agency.

Jouzel, J., Alley, R.B., Cuffey, K.M., Dansgaard, W., Grootes, P., Hoffmann, G., Johnsen, S.J., Koster, R.D., Peel, D.A., Shuman, C.A., Stievenard, M., Stuiver, M. \& White, J.W.C. 1997. Validity of the temperature reconstruction from water isotopes in ice cores. Journal of Geophysical Research, 102(C12): 26,471-26,487.

Lorius, C. \& Merlivat, L. 1977. Distribution of mean surface stable isotope values in East Antarctica: Observed changes with depth in a coastal area. IAHS Publications, 118: 125-137.

Marques, M.M., Peralba, M.C.R., Simões, J.C., Bernardo, R.T., Sneed, S.B. \& Casassa, G. 2014. Analysis of an Antarctic ice core by ion chromatography $(\mathrm{Mg}, \mathrm{Na}$, $\mathrm{Cl}$, and SO content). Geochimica Brasiliensis, 28(1): 89-96.

Masson-Delmotte, V., Hou, S., Ekaykin, A., Jouzel, J., Aristarain, A., Bernardo, R.T., Bromwich, D., Cattani, O., Delmotte, M., Falourd, S., Frezzotti, M., Galle, H., Genoni, L., Isaksson, E., Landais, A., Helsen, M.M., Hoffman, G., Lopez, J., Morgan, V., Motoyama, H., Noone, D., Oerter, H., Petit, J.R., Royer, A., Uemura, R., Schmidt, G.A., Schlosser, E., Simões, J.C., Steig, E.J., Stenni, B., Stievenard, M., Van Den Broeke, M.R., Van De Wal, R.S.W., Van De Berg, W.J., Vimeux, F. \& White, J.W.C. 2008. A review of antarctic surface snow isotopic composition: Observations, atmospheric circulation, and isotopic modeling. Journal of Climate, 21(13): 3359-3387.

Mayewski, P.A., Frezzotti, M., Bertler, N., Van Ommen, T., Hamilton, G., Jacka, T.H., Welch, B., Frey, M., Dahe, Q., Jiawen, R., Simões, J.C., Oerter, H., Nishio, F., Isaksson, E., Mulvaney, R., Holmund, P., Lipenkov, V. \& Goodwin, I. 2005. The International Trans-Antarctic Scientific Expedition (ITASE): An overview. Annals of Glaciology Fily, M., 41: 180-185.

Mayewski, P.A. \& Goodwin, D. 1997. International Trans-Antarctic Scientific Expedition (ITASE) - « 200 years of past Antarctic and Environmental Change ». Science and Implementation Plan - Report from the ITASE Workshop.

Monaghan, A.J., Bromwich, D.H. \& Wang, S.H. 2006. Recent trends in Antarctic snow accumulation from Polar MM5 simulations. Philosophical transactions. Series A, Mathematical, physical, and engineering sciences, 364(1844): 1683-1708.

Neumann, T.A. \& Waddington, E.D. 2004. Effects of firn ventilation on isotopic exchange. Journal of Glaciology, 169: 183-194.

Osterberg, E.C., Handley, M.J., Sneed, S.B., Mayewski, P.A. \& Kreutz, K.J. 2006. Continuous ice core melter system with discrete sampling for major ion, trace element, and stable isotope analyses. Environmental Science and Technology, 40(10): 3355-3361. 
Petit, J.R., Jouzel, J., Raunaud, D., Barkov, N.I., Barnola, J.M., Basile, I., Benders, M., Chappellaz, J., Davis, M., Delaygue, G., Delmotte, M., Kotlyakov, V.M., Legrand, M., Lipenkov, V.Y., Lorius, C., Pépin, L., Ritz, C., Saltzman, E. \& Stievenard, M. 1999. Climate and atmospheric history of the past 420,000 years from the Vostok ice core, Antarctica. Nature, 399: 429-436.

Qin, D., Petit, J.R., Jouzel, J. \& Stievenard, M. 1994. Distribution of stable isotopes in surface snow along the route of the 1990 International Trans-Antarctica Expedition. Journal of Glaciology, 40(134): 107-118.

Robin, G. de Q. 1983. The Climatic Record in Polar Ice Sheets. Cambridge, Cambridge University Press.

Rolph, G.D. 2003. Real-time Environmental Applications and Display sYstem (READY). NOAA Air Resour. Lab., Silver Spring, Md. Disponível em <https://www.ready.noaa.gov/>. Acesso em 08/02/2013.

\section{Man 519}

Editores: Ulisses F. Bremmer \& Paulo A. Souza.
Scambos, T.A., Frezzotti, M., Haran, T., Bohlander, J., Lenaerts, J.T.M, Van Den Broeke, M.R., Jezek, K., Long, D., Urbini, S., Farness, K., Neumann, T., Albert, M. \& Winther, J.G. 2012. Extent of low-accumulation « wind glaze " areas on the East Antarctic plateau: Implications for continental ice mass balance. Journal of Glaciology, 58(210): 633-647.

Urbini, S., Frezzotti, M., Gandolfi, S., Vincent, C., Scarchilli, C., Vittuari, L. \& Fily, M. 2008. Historical behaviour of Dome $\mathrm{C}$ and Talos Dome (East Antarctica) as investigated by snow accumulation and ice velocity measurements. Global Planet Change, 60(3-4): 576588.

Wang, Y. \& Hou, S. 2009. A new interpolation method for Antarctic surface temperature. Progress in Natural Science, 19(12): 1843-1849. 